\title{
Current Efficacy of the First Line Uncomplicated Malaria Treatment in Two Sentinels Sites of Côte d'Ivoire
}

Offianan Andre Toure ${ }^{*}$, N'Guessan Tiacoh Landry ${ }^{1}$, Ira Bonouman Ama Valerie ${ }^{1}$, Assi Serge Brice ${ }^{2}$, Kouame Emmanuel ${ }^{1}$, Kokora Adonis $^{1}$, Yao Serge Stephane ${ }^{1}$, Amata Eric ${ }^{3}$, Kinifo-Lawson Viviane ${ }^{4}$, Adji Eric Gbessi ${ }^{1}$, Beourou Sylvain ${ }^{1}$, Tuo Karil ${ }^{1}$, Ako Ako Aristide Berenger $^{1}$, Gnondjui Albert ${ }^{1}$, Djaman Joseph ${ }^{5,6}$ and Jambou Ronan ${ }^{1,7}$

${ }^{1}$ Unité de Paludologie, Institut Pasteur Côte d'Ivoire, Côte d'Ivoire

${ }^{2}$ Institut Pierre Richet Bouake/Programme National de Lutte contre le Paludisme, Abidjan Côte d'Ivoire, Côte d'Ivoire

${ }^{3}$ Formation Sanitaire Urbaine à Base Communautaire d'Anonkoua-Kouté Abidjan, Côte d'Ivoire

${ }^{4}$ Centre de Santé Urbain de Libreville, Man, Côte d'Ivoire

${ }^{5}$ Département de Biochimie Clinique et Fondamentale, Institut Pasteur Côte d'Ivoire

${ }^{6}$ Laboratoire de Pharmacodynamie-Biochimique UFR Biosciences, Université Félix Houphouët-Boigny Abidjan, Côte d'Ivoire ${ }^{7}$ Département de Parasitologie et Insectes vecteurs, Institut Pasteur Paris France, France

\begin{abstract}
Background: Artemisinin combination therapies have been wildly used in the treatment of uncomplicated falciparum malaria in most endemic countries. This strategy has been implemented in Côte d'Ivoire since 2005 with Artesunate + Amodiaquine (AS + AQ) and Artemether + lumefantrine (AL). The goal of this study was to assess efficacy and safety of these two drugs in two sentinel's sites, Man and Abidjan in Côte d'Ivoire.
\end{abstract}

Methods: An open label, randomized, clinical trial was conducted in Man in the west and Abidjan in the south of Côte d'Ivoire. Patients older than 6 months with uncomplicated falciparum malaria after consent were randomized in $\mathrm{AS}+\mathrm{AQ}$ and $\mathrm{AL}$ group and were followed up for 42 days.

The first endpoint was Adequate Clinical and Parasitological Response adjusted by PCR at day 42 . The second endpoints were fever and parasite clearance time, crude cure rate at day 42 and safety of the two ACTs.

Results: A total of 241 patients were randomized in AS+AQ (120) and AL (121) group. The crude cure rate at day 42 in $\mathrm{PP}$ analysis was $95.8 \%$ and $87.9 \%$ in AS+AQ and AL respectively. After correction by PCR ACPR at day 42 was $99.2 \%$ in AS+AQ group and $97.4 \%$ in AL group. The two ACTs were well tolerated.

Conclusion: $\mathrm{AS}+\mathrm{AQ}$ and $\mathrm{AL}$ remains efficacious in the uncomplicated malaria treatment in the two areas but continue monitoring is needed particularly for AL.

\section{Background}

Malaria remains a major public health problem instead of many strategies developed by WHO and his partners to control and eliminate this disease. In malaria endemic Sub-Saharan countries pregnant women and children under-fives years are a high risk of malaria and death. Malaria control programs currently comprise integrated interventions, including vector control with use of mosquito nets and spraying with insecticides, Intermittent Preventive Treatment in pregnancy (IPT), early diagnosis and effective treatment of clinical cases [1]. Artemisinin Combination Therapy (ACT) associated with others control and elimination strategies are contributed to reduced malaria morbidity and mortality. But emergence and extension of resistance to artemisinins derivatives in South East Asia could counteract these benefits [1]. Currently, Artemether + Lumefantrine (AL) and Artesunate + Amodiaquine (AS-AQ) are the widely available drugs, which are recommended by most malaria endemic countries in the treatment of uncomplicated falciparum malaria [2]. Monitoring of artemisinin-based combination therapy (ACT) becomes particularly important in the light of emergence of artemisinin resistance in South-East Asia [3,4].

The World Health Organization (WHO) recommends that the efficacy of the first and second-line antimalarial drugs be regularly assessed for early detection and prevention of spread of resistant parasite populations every two years [5]. efficacious and well tolerated [7]. org/10.15344/2456-8007/2018/124

\section{Publication History:}

Received: October 19, 2017

Accepted: March 01, 2018

Published: March 03, 2018

\section{Keywords:}

Malaria falciparum ACTs Côte d'Ivoire studies to measure early clearance of peripheral parasitaemia by microscopy and k13 propeller gene mutations [6]. In Côte d'Ivoire, $\mathrm{AS}+\mathrm{AQ}$ and $\mathrm{AL}$ have been recommended in the treatment of uncomplicated malaria since 2005. To detect early artemisinin resistance in the country National Malaria Control Program (NMCP) of Côte d'Ivoire conducted with the help of his Scientific Committee efficacy studies of ACTs recommended for uncomplicated falciparum malaria treatment in the country every two years since 2009. Twelve sentinels sites have been identified around the country and Abidjan and Man are among the first sites since several years. Previous study conducted in these two sites in 2013 demonstrated that both ACT was

The main purpose of this study was to assess efficacy and safety of AS $+\mathrm{AQ}$ and AL the two recommended ATCs in the first line treatment of uncomplicated falciparum malaria in Côte d'Ivoire. Data from this study will help NMCP to update malaria treatment guidelines.

${ }^{\circ}$ Corresponding Author: Dr. Offianan Andre Toure, Unité de Paludologie, Institut Pasteur Côte d'Ivoire, Côte d'Ivoire; E-mail: andre_offianan@yahoo.fr

Citation: Toure OA, Landry NT, Valerie IBA, Brice AS, Emmanuel K, et al. (2018) Current Efficacy of the First Line Uncomplicated Malaria Treatment in Two Sentinels Sites of Côte d'Ivoire. Int J Clin Res Trials 3: 124. doi: https://doi.

Copyright: () 2018 Toure et al. This is an open-access article distributed under the terms of the Creative Commons Attribution License, which permits unrestricted use, distribution, and reproduction in any medium, provided the original author and source are credited. 
Citation: Toure OA, Landry NT, Valerie IBA, Brice AS, Emmanuel K, et al. (2018) Current Efficacy of the First Line Uncomplicated Malaria Treatment in Two Sentinels Sites of Côte d'Ivoire. Int J Clin Res Trials 3: 124. doi: https://doi.org/10.15344/2456-8007/2018/124

Page 2 of 7

\section{Materials \& Method}

\section{Study design and sites}

This study was an open label, non-inferiority, randomized trial conducted in patients older than 6 months at Man and Abidjan and was carried out according to current $\mathrm{WHO}$ protocol [5]. The study was conducted from January to May 2016 in the two sites.

Abidjan located in the south and Man in the west of the country are among the sentinels sites for antimalarial drugs efficacy monitoring since several years.

Malaria transmission is intense with recrudescence during the raining season in the two sites. The main vectors are Anopheles gambiae and Anopheles funestus. Plasmodium falciparum is the predominant malaria parasite, accounting for more than $95 \%$ of infections in children under five years of age [7]. The key malaria control interventions in the sites include use of LLNIs, malaria case management with ACTs, Intermittent Preventive Treatment during Pregnancy (IPTp).

\section{Study population}

Patients were enrolled in the study according to Who inclusion and exclusion criteria [5].Briefly patients older than 6 months with the following inclusion criteria were eligible for the study: monoinfection with P. falciparum detected by microscopy, parasite density between 2,000 and 200,000 asexual parasites/ $\mu$ l, axillary temperature $\geq 37.5^{\circ} \mathrm{C}$ or history of fever during the 24 hours before recruitment.

Additional inclusion criteria were minimum body weight of $5 \mathrm{~kg}$, ability to swallow oral medication, informed consent from the patient or from a parent or guardian in the case of children; and ability and willingness to comply with the protocol for the duration of the study and to comply with the study visit schedule.

Exclusion criteria included general danger signs in children under 5 years or signs of severe falciparum malaria according to the definitions of WHO [8]; severe malnutrition according to WHO child growth standards [9]; febrile condition due to diseases other than malaria (e.g. measles, acute lower respiratory tract infection, severe diarrhea with dehydration) or other known underlying chronic or severe diseases (e.g. cardiac, renal or hepatic diseases, HIV/AIDS).

\section{Sample size}

The sample size was calculated using the WHO guidelines on assessment of antimalarial drugs [5]. Population size was determined according to the following criteria: the proportion of probable clinical failures with the antimalarial combinations studied should not be higher than $10 \%$, for a level of confidence $(\mathrm{P})$ of $95 \%$ and a precision (p) of $10 \%$, taking into account patients who were excluded or lost to follow-up. Using these criteria, a minimum of 50 patients was required in each treatment arm in each site.

\section{Randomization and Drug administration}

Patients were randomly assigned to receive either AS+AQ or AL. Both treatments were three-day oral regimens dosed by weight according to the manufacturer's instructions: AS + AQ 5 to $<9 \mathrm{~kg}$ : one tablet/day of artesunate (AS) $25 \mathrm{mg} /$ amodiaquine (AQ) $67.5 \mathrm{mg}$; 9 to
$<18 \mathrm{~kg}$ : one tablet/day of AS $50 \mathrm{mg} / \mathrm{AQ} 135 \mathrm{mg} ; 18$ to <36 kg: 1 tablet/ day of AS $100 \mathrm{mg} / \mathrm{AQ} 270 \mathrm{mg}$; $\geq 36 \mathrm{~kg}: 2$ tablets/day of AS $100 \mathrm{mg} /$ AQ $270 \mathrm{mg}$. AS+AQ was administered once daily under observation by the research team.

AL tablet strength was $20 \mathrm{mg}$ artemether/120 mg lumefantrine: 5 to $<15 \mathrm{~kg}: 1$ tablet/dose; 15 to $<25 \mathrm{~kg}: 2$ tablets/dose; 25 to $<35 \mathrm{~kg}: 3$ tablets/dose; $\geq 35 \mathrm{~kg} 4$ tablets/dose. AL was administrated twice a day.

The first dose was taken at enrolment, the second dose eight hours later on day 0 , and then two doses at 12-hourly intervals for the subsequent two days. The first dose was given at the site by the research team. The second dose was not directly observed and patients were precisely instructed how and when to take this second dose. All tablets were either swallowed whole or crushed with water and Yoghurt.

Patients who vomited during the observation period were retreated with the same dose of medicine and observed for an additional 30 minutes. Patients with repeated vomiting were excluded and were treated according to NMCP treatment guidelines and excluded from the study.

\section{Clinical procedures}

Physical examination, vital signs, body temperature, and clinical assessment were done at day 0 (screening), and at all follow-up visits (days 1, 2, 3, 7, 14, 21, 28, 35 and 42).

Medical history, demographic information and contact details were collected at screening. We recorded body weight at date 0 and Axillary, or rectal temperature was performed at baseline (day 0 before dosing) and on days 1, 2, 3, 7, 14, 21, 28, 35 and 42 or at any unscheduled visit.

\section{Laboratory Procedures}

\section{Microscopy}

Thick/thin blood smears were collected at the time of screening to confirm adherence to the inclusion and exclusion criteria. Thick blood films were also done at days 2, 3, 7, 14, 21, 28, (35 and 42) or on any other day if the patient returns spontaneously and parasitological reassessment is required. Blood smears were stained with Giemsa $10 \%$ and were examined at magnification $100 \mathrm{X}$ (oil immersion). Parasitemia was measured by counting the number of asexual parasites against a number of leukocytes in the thick blood film, based on a putative count of 8000 leukocytes per microliter of blood.

The number of asexual parasites was counted against 200 leukocytes using a hand tally counter. If P. falciparum gametocytes were seen, a gametocyte count was performed against 1000 leukocytes. Counts were performed by two independent microscopists; discrepant readings were resolved by a third reader.

\section{Parasite genotyping}

Blood spots on Whatman $3 \mathrm{MM}$ filter paper were prepared for PCR genotyping on screening and in the event of re-appearance of parasites, confirmed by microscopy, or on any other day if a patient returned with fever. Multiplicity of infection was assessed using three polymorphic loci (merozoite surface proteins 1 and 2 and glutamate-rich protein). Briefly, filter paper blood samples collected 
Citation: Toure OA, Landry NT, Valerie IBA, Brice AS, Emmanuel K, et al. (2018) Current Efficacy of the First Line Uncomplicated Malaria Treatment in Two Sentinels Sites of Côte d'Ivoire. Int J Clin Res Trials 3: 124. doi: https://doi.org/10.15344/2456-8007/2018/124

Page 3 of 7

on the day of enrolment and on the day of failure were analysed for polymorphism of merozoite surface protein-1 (msp-1) and merozoite surface protein-2 ( $m s p-2)$ using nested-PCR as previously described [10]. Possible outcomes were new infection or recrudescence. A "new infection" is a subsequent occurring parasitaemia in which all the alleles in parasites from the post-treatment sample are different from those in the admission sample, for one or more loci tested. In a "recrudescence," at least one allele at each locus should be in common for both paired samples.

\section{Statistical analysis}

Data generated were recorded in individual participants Case Record form (CRF). Data were entered and analyzed with EPI-Info ${ }^{\text {Tm }}$ software (v 7.1; Centers for Disease Control and Prevention, Atlanta GA). Analysis of treatment outcome was intention-to-treat (ITT) and per protocol. Per-protocol analysis included all patients who matched all inclusion criteria, were properly randomized, had received the study drugs according to the protocol, and for whom data were available on the study endpoint without protocol violation during the follow-up time. ITT analysis comprised all randomized patients fulfilling the inclusion criteria without repeated vomiting after the first study drug administration. Frequencies were compared by either chi-squared or Fisher's exact tests, as appropriate, and continuous variables by Student's t-tests when the data are normally distributed.

\section{Ethical issues}

The study was conducted according to the Declaration of Helsinki and national legal and regulatory requirements. Protocol, CRF and consent form were approved by the "Comité National d'Ethique de la Recherche" of Côte d'Ivoire.

\section{Results}

\section{Study trial profile and baseline characteristics}

A total of 1,188 patients have been screened for eligibility criteria in the two sites among them 120 and 121 were randomized respectively in $\mathrm{AS}+\mathrm{AQ}$ and $\mathrm{AL}$ group.

A total of 118 and 116 patients had available data for PP analysis at day 42 respectively in AS+AQ and AL group (Figure 1).The patient population had $56(46.7 \%)$ male and $64(53.3 \%)$ female patients in AS+AQ group. In AL group patient population had 62 (51.2\%) and $59(48.8 \%)$ males and female respectively. The mean age was 8.7 and 11.1 years respectively in $\mathrm{AS}+\mathrm{AQ}$ and $\mathrm{AL}$ group. Overall, both groups of patients were comparable at the inclusion regarding baseline characteristics (Table1).

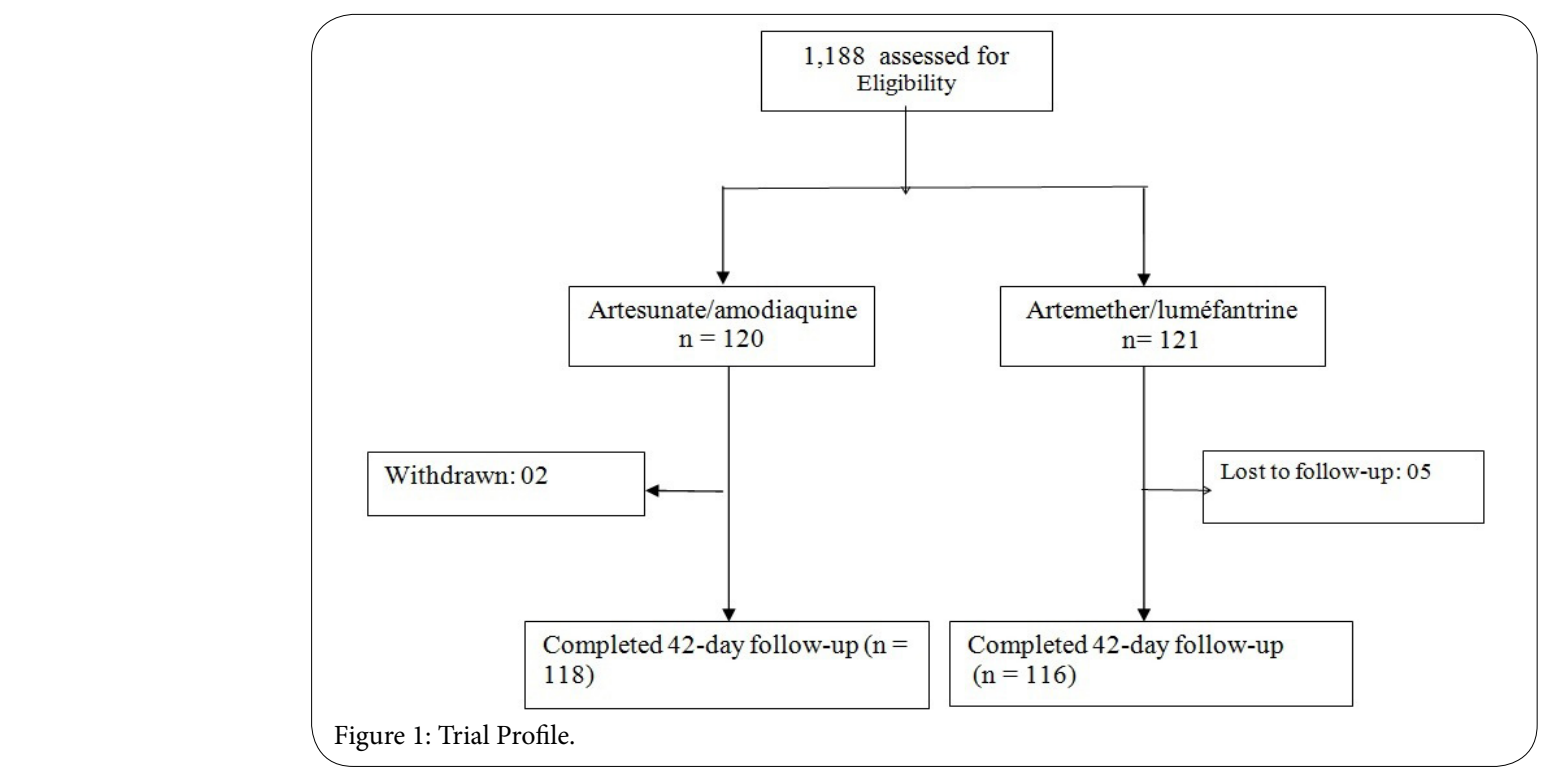

\begin{tabular}{|l|l|l|l|}
\hline & ASAQ $(\mathrm{N}=120)$ & AL $(\mathrm{N}=121)$ & \\
\hline Male $(\%)$ & $56(46.7)$ & $62(51.2)$ & $0,60 \mathrm{a}$ \\
\hline Female $(\%)$ & $64(53.3)$ & $59(48.8)$ & $0.60 \mathrm{a}$ \\
\hline Mean Age (years) $( \pm \mathrm{SD})$ & $8.7(9.92)$ & $11.1(12.48)$ & $0.11 \mathrm{~b}$ \\
\hline$<5$ years $(\%)$ & $49(40.8)$ & $52(43)$ & $0.89 \mathrm{a}$ \\
\hline $5-<15$ years $(\%)$ & $51(42.5)$ & $41(33.9)$ & $0.23 \mathrm{a}$ \\
\hline$\geq 15$ yeras $(\%)$ & $20(16.7)$ & $28(23.1)$ & $0,25 \mathrm{a}$ \\
\hline Mean Temperature $\left({ }^{\circ} \mathrm{C}\right)( \pm \mathrm{SD})$ & $38.3(0.74)$ & $38.1(0.61)$ & $0.09 \mathrm{~b}$ \\
\hline Mean Weight $(\mathrm{Kg})( \pm \mathrm{SD})$ & $25(20.12)$ & $29.2(23.09)$ & $0.13 \mathrm{~b}$ \\
\hline Mean Parasitemia $($ asexual parasites/ $\mu \mathrm{l})( \pm \mathrm{SD})$ & $40112(49235)$ & $31479(40541)$ & $0,13 \mathrm{~b}$ \\
\hline
\end{tabular}

Table 1: Participant's baseline characteristics at day 0 .

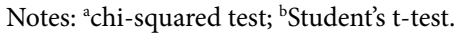

Abbreviations: AS-AQ, artesunate-amodiaquine; AL, artemether-lumefantrine; GM, geometric mean, ITT, intention to treat. 
Citation: Toure OA, Landry NT, Valerie IBA, Brice AS, Emmanuel K, et al. (2018) Current Efficacy of the First Line Uncomplicated Malaria Treatment in Two Sentinels Sites of Côte d'Ivoire. Int J Clin Res Trials 3: 124. doi: https://doi.org/10.15344/2456-8007/2018/124

Page 4 of 7

\section{Fever and parasite clearance}

As shown in Figure 2, the proportion of participants without fever was similar on days 2 and 3 . On day 2, the fever clearance rates was 99.2\% and $98.3 \%$ in the AS +AQ and AL groups, respectively (p $>0$. 5), while on day 3 , this rate was $100 \%$ in each group (Figure 2 ). Both treatments resulted in rapid clearance of parasites (Figure 3) as parasite clearance rates on day 2 was $95 \%$ and $90.7 \%$ in the AS + AQ and AL groups, respectively. At day 3 parasite clearance rate was $100 \%$ in AS+AQ group and $97.4 \%$ in AL group. 4 patients presented delayed parasite clearance at day 3 in AL group.

\section{Therapeutic Response and tolerability of drugs}

The PCR-corrected ACPR at day 42 in PP analysis was $99.2 \%$ and 97.4 for AS+AQ and AL, respectively. In the ITT analysis, the irrespective ACPR was $97.5 \%$ for AS + AQ and $93.4 \%$ for AL (Table 2). Most treatment failure (10 patients) occurred in AL group at Abidjan site among them 9 patientshave taken ALfrom the same Pharmaceuticallab.
The most commonly reported and possibly drug-related adverse events to both combination therapies were effects of malaria infection. None of the patients presented with serious adverse events. Adverse effects such as anorexia ( $42.5 \%$ vs $28.1 \%$ ), drowsiness ( $28 \%$ vs $0 \%$ ), dizziness ( $30 \%$ vs $2.5 \%)$, Headache $(77.7 \%$ vs $48.8 \%$ ) were more frequent in the AS+AQ group compared to the AL group ( $\mathrm{p}<0.05)$. All of these events were described as mild or moderate (Table 3 ).

\section{Discussion}

Malaria is still associated with high morbidity and mortality especially in children under five years of age in Sub-Saharan endemic countries.

Artemisinin-based combination therapies (ACTs) are recommended by most of National malaria control program as the first-line treatment for uncomplicated P. falciparum malaria. This strategy, has, produced a notable decrease in the mortality and in reducing the global malaria

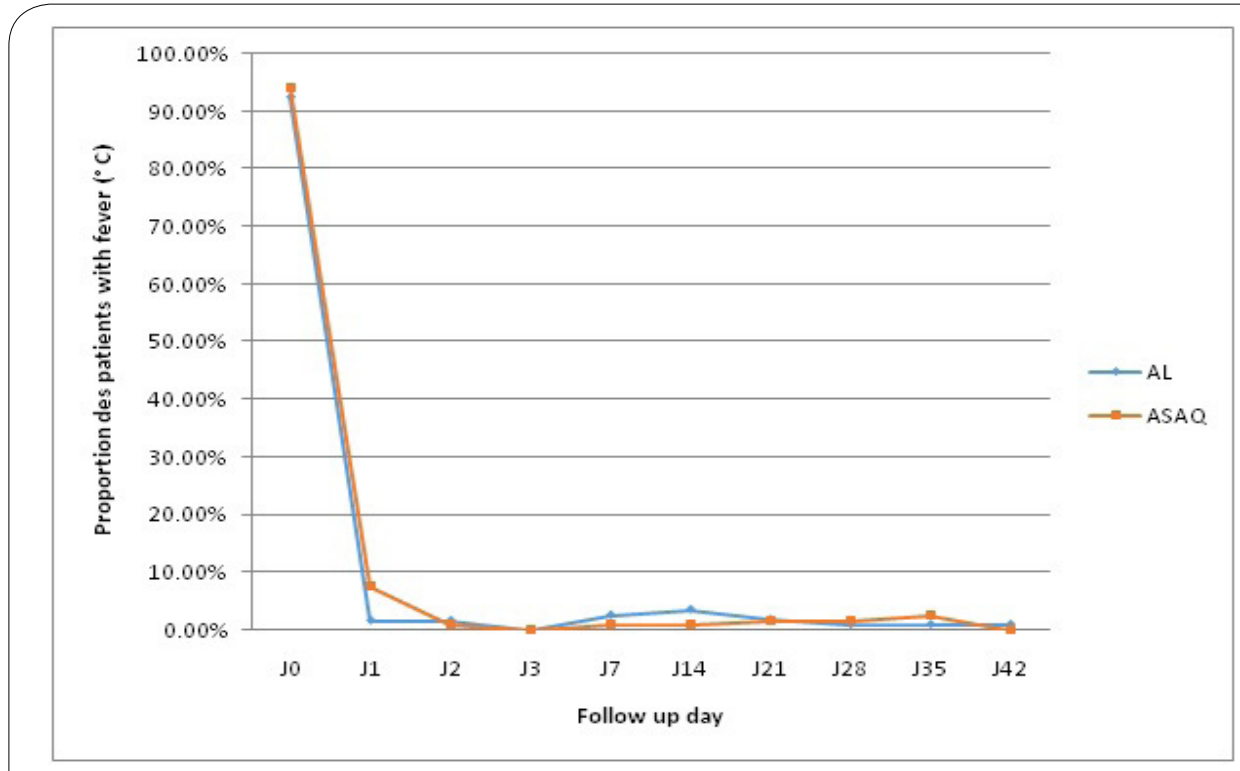

Figure 2: Proportion of patients with fever during follow up.

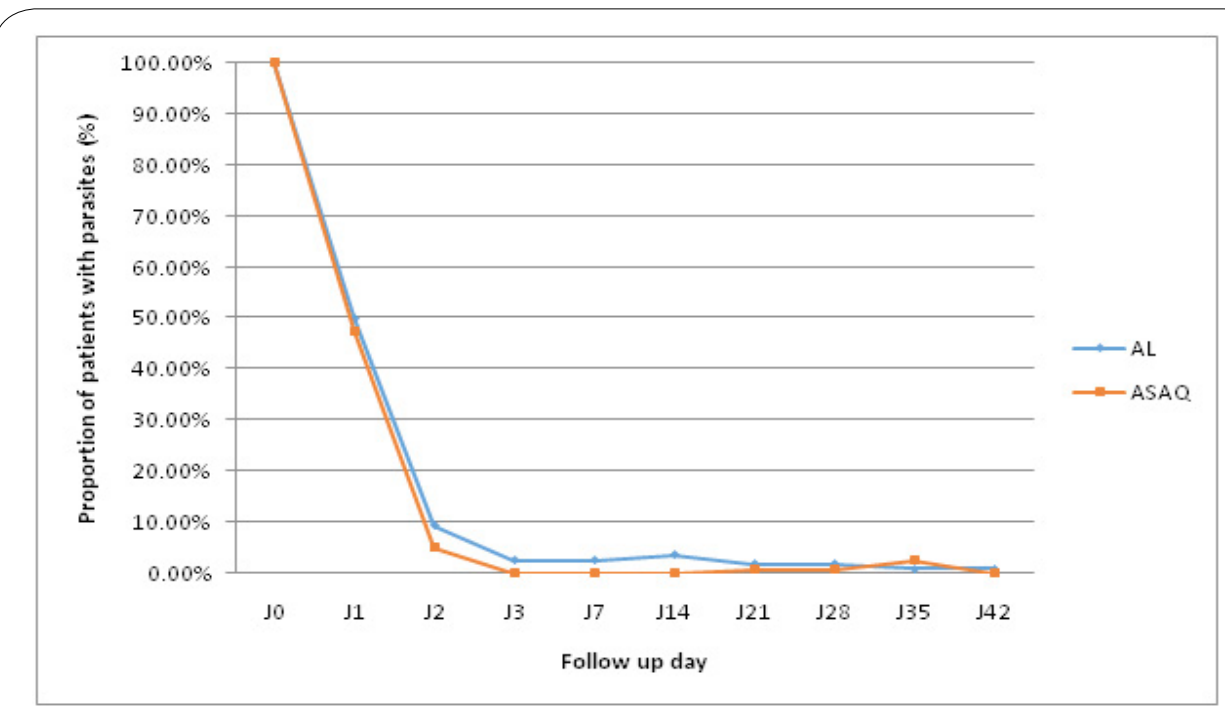

Figure 3: Proportion of patients with parasites during follow up. 
Citation: Toure OA, Landry NT, Valerie IBA, Brice AS, Emmanuel K, et al. (2018) Current Efficacy of the First Line Uncomplicated Malaria Treatment in Two Sentinels Sites of Côte d'Ivoire. Int J Clin Res Trials 3: 124. doi: https://doi.org/10.15344/2456-8007/2018/124

Page 5 of 7

burden. However, Resistance of Plasmodium to artemisinins reported in several countries in South-East Asiais, therefore, of great concern $[11,12,13]$. The spread or independent emergence of artemisinin resistance in other parts of the world could pose a major public health problem as no alternative antimalarial medicine is available at present with the same level of efficacy and tolerability as ACTs.

The present study based on the in vivo efficacy using 42 -follow up days protocol was conducted to provide supporting evidence for the clinical efficacy of AS + AQ and AL in the country. The two ACTs were adopted and implemented as anti-malarial drug policy in Côte d'Ivoire since 2005. Results from this study demonstrated high efficacy of the two ACTs as PCR adjusted ACPR for each ACT remains above the 95 $\%$ efficacy threshold recommended by the WHO [5].Both treatments proved to be efficacious but delayed parasite clearance time observed in $\mathrm{AL}$ group in Abidjan site raise concerns. In this study more than 3 trade name of artemether + lumefantrine have been used at Abidjan site. All these drugs have been provided by the NMCP. Among 10 patients with clinical failure 9 have received the $\mathrm{AL}$ drug from the same pharmaceutical lab. Administration of artemether-lumefantrine with food is important to achieve sufficient exposure to lumefantrine up to day 7 , which is required for a high cure rate $[14,15]$. In this study only first doses of AL were observed. As absorption is fat dependent, patients were precisely instructed to give the second dose with fatty food at home. However, intake of this second dose was not directly controlled. Food is known to increase the bioavailability (BA) of lumefantrine by up to 16 -fold and that of artemether by up to 3 -fold.

General inter-individual variability for drug absorption and pharmacokinetic may be associated with changes in the bioavailability of a drug and consequently may affect the therapeutic efficacy.

A study conducted in Nigeria indicated an increase in parasite clearance time and high frequency of crude late parasitological

\begin{tabular}{|l|l|l|l|}
\hline Outcome & ASAQ $(\%)$ & AL $(\%)$ & p-value \\
\hline IIT Analysis & 120 & 121 & \\
\hline Enrolled patients & $118 / 120(98.3)$ & $116 / 121(95.9)$ & 0.44 \\
\hline Patients seen & $02 / 120(1.7)$ & $05 / 121(4.1)$ & 0.44 \\
\hline Missing & $07 / 120(5.8)$ & $19 / 121(15.7)$ & 0.05 \\
\hline Crude failure rate & $113 / 120(94.2)$ & $102 / 121(84.3)$ & 0.17 \\
\hline Crude cure rate & $03 / 120(2.5)$ & $8 / 121(6.6)$ & 0.22 \\
\hline PCR adjusted failure rate & $115 / 120(97.5)$ & $113 / 121(93.4)$ & 0.57 \\
\hline PCR adjusted ACPR & \multicolumn{3}{|l|}{} \\
\hline PP Analysis & 118 & 116 & \\
\hline Patients seen & $05 / 118(4.20)$ & $14 / 116(12.1)$ & 0.05 \\
\hline Crude failure rate & $113 / 118(95.8)$ & $102 / 116(87.9)$ & 0.05 \\
\hline Crude cure rate & $01 / 118(0.8)$ & $03 / 116(2.6)$ & 0.60 \\
\hline PCR adjusted failure rate & $117 / 118(99.2)$ & $113 / 116(97.4)$ & 0.60 \\
\hline PCR adjusted ACPR & &
\end{tabular}

Table 2: Therapeutic response in the two groups at day 42 .

$\mathrm{AS}+\mathrm{AQ}$, artesunate + amodiaquine; $\mathrm{AL}$, artemether-lumefantrine. $\mathrm{P}$ values were obtained by Fisher's exact test.

\begin{tabular}{|c|c|c|c|c|c|c|c|c|c|}
\hline & \multicolumn{4}{|c|}{ ASAQ $(\mathrm{N}=120)$} & \multicolumn{4}{|c|}{$\mathrm{AL}(\mathrm{N}=121)$} & \multirow{2}{*}{$\begin{array}{l}\text { Fisher's Exact Test } \\
\text { p-value }\end{array}$} \\
\hline & $\mathrm{n}$ & $\%$ & $95 \%$ & IC & $\mathrm{n}$ & $\%$ & $95 \%$ & IC & \\
\hline Anorexia & 51 & 42.5 & 28.0 & 57.0 & 34 & 28.1 & 11.5 & 44.7 & 0.03 \\
\hline Asthenia & 43 & 35.8 & 20.3 & 51,3 & 35 & 28.9 & 12.5 & 45.4 & 0.33 \\
\hline Nausea & 14 & 11.8 & -8.7 & 32.1 & 8 & 6.6 & -16.9 & 30.1 & 0.26 \\
\hline drowsiness & 33 & 27.5 & 10.8 & 44.2 & 0 & 0 & - & - & $<0.00001$ \\
\hline Cough & 2 & 01.7 & -41.1 & 44.4 & 12 & 9.9 & -11.2 & 31.0 & 0.01 \\
\hline Dizziness & 36 & 30.0 & 13.6 & 46.4 & 3 & 2.5 & -31.8 & 36.7 & $<0.00001$ \\
\hline Vomiting & 25 & 20.8 & 2.9 & 38.8 & 16 & 13.2 & -6.5 & 32.9 & 0.17 \\
\hline Headache & 86 & 71.7 & 61.6 & 81.8 & 59 & 48.8 & 35.2 & 62.4 & $<0.00001$ \\
\hline Myalgia & 24 & 20.0 & 1.9 & 38.1 & 22 & 18.2 & -0.2 & 36.6 & 0,86 \\
\hline Arthralgia & 34 & 28.3 & 11.7 & 45.0 & 34 & 28.1 & 11.5 & 44.7 & 1 \\
\hline Diarrhea & a & 04.2 & -23.3 & 31.7 & 5 & 4.1 & -23.3 & 31.6 & 1 \\
\hline Pruritus & 6 & 05.0 & -20.8 & 30.8 & 3 & 2.5 & -31.8 & 36.7 & 0.49 \\
\hline Abdominal pain & 10 & 08.3 & -13.8 & 30.5 & 5 & 4.1 & -23.3 & 3.6 & 0.28 \\
\hline
\end{tabular}

Table 3 : Clinical adverse events. 
Citation: Toure OA, Landry NT, Valerie IBA, Brice AS, Emmanuel K, et al. (2018) Current Efficacy of the First Line Uncomplicated Malaria Treatment in Two Sentinels Sites of Côte d'Ivoire. Int J Clin Res Trials 3: 124. doi: https://doi.org/10.15344/2456-8007/2018/124

Page 6 of 7

failures among the patients, indicating reduction in the therapeutic efficacy of Artemether + lumefantrine [16].

In the same site (Abidjan) in 2013, three years ago ACPR after adjustment by PCR of AS + AQ and AL was $100 \%$ for each drug at day 42.The same result was obtained with the two ACTs in Man site in 2013 [7]. Others studies conducted in the country have demonstrated also good efficacy of AS+AQ and AL in the treatment of uncomplicated falciparum malaria $[17,15,19-22]$.

Although most studies carried out in malaria endemic countries in Africa [23-26] had shown good efficacy and safety of ACTs for the treatment of uncomplicated malaria, the conditions of clinical trials do not fully reflect real field situation. Results from studies conducted with unsupervised malaria treatment showed low cure rate after adjustment by PCR at day $28[27,28]$.

Some studies demonstrated that unsupervised intake of $\mathrm{AL}$ was highly efficacious despite the lumefantrine plasma levels concentrations being significantly lower than in patients whose treatment had been supervised [29-32].

Both treatments were generally well tolerated; the overall incidences of AEs were more frequent in the AS + AQ than AL treatment groups. The most commonly occurring AEs included anorexia, drowsiness, dizziness and Headache.

Our study has several limitations. The lack of sufficient sampling times for fever and parasite clearance time. We were not able to determine the levels of drugs concentration which may have been of interest for its contribution to the therapeutic efficacy of ACTs.

\section{Conclusion}

Although results from this study proved good efficacy of AS+AQ ad $\mathrm{AL}$ in the treatment of uncomplicated falciparum malaria in the two sites, constant monitoring of these two ACTs is needed.

\section{Competing Interests}

The authors declare that they have no competing interests.

\section{Author's Contributions}

All authors participated in the design, implementation, and analysis or data interpretation of the study. Offianan AT was involved in all phases of the study, N'guessan Tiacoh L, Kouame E, Kokora A., Amata Eric, Kinifo-Lawson V were responsible for conducting field studies and coordination of study procedures. Ira Bonouma, Djaman Jisephn Assi Serge B and Ronan jambou supervised the study. Data analysis was done by Adji Eric Gbessi. Coulibaly Baba and Ako Ako Aristide B performed PCR analysis. Yao Serge S and Albert Gnondjui undertook lab procedures. The manuscript was drafted by Offianan Andre Toure. All authors critically reviewed the report and approved the final version of the report for submission.

\section{Acknowledgments}

We are grateful to participants, medical staff and all health authorities in the study sites for collaboration. The authors would also like to acknowledge National malaria Control Program and WHO Global Fund.

\section{Funding}

The National Malaria Control Program of Côte d'Ivoire and WHO Global Fund sponsored this trial.

\section{References}

1. World Health Organization (2016) World malaria report.

2. World Health Organization (2015) Guidelines for the treatment of malaria. 3nd edition. Geneva: WHO Press.

3. Dondorp AM, Yeung S, White L, Nguon C, Day NP, et al. (2010) Artemisinin resistance: current status and scenarios for containment. Nat Rev Microbiol: 8: $272-280$.

4. Phyo AP, Nkhoma S, Stepniewska K, Ashley EA, Nair S, et al. (2012) Emergence of artemisinin-resistant malaria on the western border of Thailand: a longitudinal study. Lancet 379: 1960-1966.

5. World Health Organization (2009) Methods for surveillance of antimalarial drug efficacy. Geneva: World Health Organization.

6. Ariey F, Witkowsky B, Amaratunga C, Beghain J, Langlois AC, et al. (2014) A molecular marker of artemisinin resistant Plasmodium falciparum. Nature 505: 50-55

7. Offianan AT, Assi SB, Tiacoh LN, Gbessi EA, Ako AB, et al. (2014) Open label, randomized, non-inferiority clinical trial of artesunate-amodiaquine versus artemether-lumefantrine fixed-dose combinations in children and adults with uncomplicated falciparum malaria in Cote d'Ivoire. Malar J 13: 439.

8. WHO (2000) Severe falciparum malaria. Transactions of the Royal Society of Tropical Medicine and Hygiene.

9. $\mathrm{WHO}(2006 \mathrm{~b}) \mathrm{WHO}$ child growth standards: length/height-for-age, weightfor-age, weight-for-length, weight-for-height and body mass index-forage: methods and development. Geneva: World Health Organization Multicentre Growth Reference Study Group.

10. MMV/WHO (2007) Methods and techniques for clinical trials on antimalarial drug efficacy: genotyping to identify parasite populations. Amsterdam, The Netherlands: WHO.

11. Noedl H, Socheat D, Satimai W (2009) Artemisinin-resistant malaria in Asia. $N$ Engl J Med 361: 540-541.

12. Dondorp AM, Nosten F, Yi P, Das D, Phyo AP, et al. (2009) Artemisinin resistance in Plasmodium falciparum malaria. N Engl J Med 361: 455-467.

13. Nyunt MH, Soe MT, Myint HW, Oo HW, Aye MM, et al. (2017) Clinical and molecular surveillance of artemisinin resistant falciparum malaria in Myanmar (2009-2013). Malar J 16: 333.

14. Ashley EA, Stepniewska K, Lindegårdh N, McGready R, Annerberg A, et al (2007) Pharmacokinetic study of artemether-lumefantrine given once daily for the treatment of uncomplicated multidrug-resistant falciparum malaria. Trop Med Int Health 12: 201-208.

15. World Wide Antimalarial Resistance Network (WWARN) Lumefantrine PK/PD Study Group (2015) Artemether-lumefantrine treatment of uncomplicated Plasmodium falciparum malaria: a systematic review and meta-analysis of day 7 lumefantrine concentrations and therapeutic response using individual patient data. BMC Med 13: 227.

16. Ayogu EE, Ukwe CV, Nna EO (2015) Therapeutic Efficacy of ArtemetherLumefantrine for Treatment of Uncomplicated Plasmodium falciparum Malaria in Enugu, Nigeria. Tropical Journal of Pharmaceutical Research; 14: 1487-1493.

17. Menan H, Faye O, Same-Ekobo A, Oga ASS, Faye B, et al. (2011) Comparative study of the efficacy and tolerability of dihydroartemisininpiperaquine-trimethoprim versus artemether-lumefantrine in the treatment of uncomplicated Plasmodium falciparum malaria in Cameroon, Ivory Coast and Senegal. Malar J 10: 185.

18. Offianan AT, Menan GK, Yapi JD, Ako AAB, Khali D, et al. (2011) Artesunate/ mefloquine paediatric formulation vs. artemether/lumefantrine for the treatment of uncomplicated Plasmodium falciparum in Anonkoua koute, Côte d'Ivoire. Trop Med Int Health, 16: 290-297.

19. Toure Offianan A, Assi SB, Aristide MA C, N'guessan LT, Ako AA, et al. (2011) Assessment of the efficacy of first-line antimalarial drugs after 5 years of deployment by the National Malaria Control Programme in Côte d'Ivoire. Open Access Journal of Clinical Trials, 3: 67-76. 
Citation: Toure OA, Landry NT, Valerie IBA, Brice AS, Emmanuel K, et al. (2018) Current Efficacy of the First Line Uncomplicated Malaria Treatment in Two Sentinels Sites of Côte d'Ivoire. Int J Clin Res Trials 3: 124. doi: https://doi.org/10.15344/2456-8007/2018/124

Page 7 of 7

20. Faye B, Kuété T, Kiki-Barro CP, Tine RC, Nkoa T, et al. (2012) Multicentre study evaluating the non-inferiority ofthe new paediatric formulation of artesunate/amodiaquine versus artemether/lumefantrine for the management of uncomplicated Plasmodium falciparum malaria in children in Cameroon, Ivory Coast and Senegal. Malar J 11: 433.

21. Toure OA, Valecha N, Tshefu AK, Thompson R, Krudsood S, et al. (2016) A Phase 3, Double-Blind, Randomized Study of Arterolane MaleatePiperaquine Phosphate vs Artemether-Lumefantrine for Falciparum Malaria in Adolescent and Adult Patients in Asia and Africa. Clinical Infectious Diseases 62: 964-971.

22. Yavo W, Konaté A, Kassi FK, Djohan V, Angora KE, et al. (2015) Efficacy and Safety of Artesunate-Amodiaquine versus Artemether Lumefantrine in the Treatment of Uncomplicated Plasmodium falciparum Malaria in Sentine Sites across Côte d'Ivoire. Malar Res Treat 2015: 878132

23. Dorkenoo AM, Yehadji D, Agbo YM, Layibo Y, gbeko F, et al. (2016) Therapeutic efficacy trial of artemisinin-based combination therapy for the treatment of uncomplicated malaria and investigation of mutations in $\mathrm{K} 13$ propeller domain in Togo, 2012-2013. Malar J 15: 331.

24. Ogouyèmi-Hounto $A$, Azandossessi $C$, Lawani $S$, Damien $G$, SissintoSavi de Tove Y, et al. (2016) Therapeutic efficacy of artemether-lumefantrine for the treatment of uncomplicated falciparum malaria in northwest Benin. Malar J 15: 37.

25. Plucinski MM, Dimbu PR, Macaia AP, Ferreira CM, Samutondo C, et al. (2017) Efficacy of artemether-lumefantrine, artesunate-amodiaquine, and dihydroartemisinin-piperaquine for treatment of uncomplicated Plasmodium falciparum malaria in Angola, 2015. Malar J 16: 62.

26. Salvador C, Rafael B, Matsinhe F, Candrinho B, Muthemba R, et al. (2017) Efficacy and safety of artemether-lumefantrine for the treatment of uncomplicated falciparum malaria at sentinel sites in Mozambique, 2015. Acta Trop 171: 146-150.

27. Tinto H, Diallo S, Zongo I, Guiraud I, Valea I, et al. (2014) Effectiveness of artesunate-amodiaquine vs. artemether-lumefantrine for the treatment of uncomplicated falciparum malaria in Nanoro, Burkina Faso a noninferiority randomised trial. Trop Med Int Health 19: 469-475.

28. Sondo P, Derra K, Diallo-Nakanabo S, Tarnagda Z, Zampa O, et al. (2015) Effectiveness and safety of artemether-lumefantrine versus artesunateamodiaquine for unsupervised treatment of uncomplicated falciparum malaria in patients of all age groups in Nanoro, Burkina Faso: a randomized open label trial. Malar J 14: 325.

29. Mutabingwa TK, Anthony D, Heller A, Hallett R, Ahmed J, et al. (2005) Amodiaquine alone, amodiaquine+sulfadoxine-pyrimethamine amodiaquine+artesunate, and artemether-lumefantrine for outpatient treatment of malaria in Tanzanian children: a four-arm randomised effectiveness trial. Lancet 365: 1474-1480.

30. Piola P, Fogg C, Bajunirwe F, Biraro S, Grandesso F, et al. (2005) Supervised versus unsupervised intake of six-dose artemether-lumefantrine for treatment of acute, uncomplicated Plasmodium falciparummalaria in Mbarara, Uganda: a randomised trial. Lancet 365: 1467-1473.

31. Checchi F, Piola P, Fogg C, Bajunirwe F, Biraro S, et al. (2006) Supervised versus unsupervised antimalarial treatment with six-dose artemetherlumefantrine: pharmacokinetic and dosage-related findings from aclinical trial in Uganda. Malaria Journal 5: 59.

32. Ngasala BE, Malmberg $M$, Carlsson AM, Ferreira PE, Petzold MG, et al (2011) Effectiveness of artemether-lumefantrine provided by community health workers in under-five children with uncomplicated malaria in rural Tanzania: an open label prospective study. Malaria Journal 10: 64 . 